\title{
Assessing the Integration of Electrified On- Board Systems in an MDAO framework for a small transport aircraft
}

\author{
M. Fioriti ${ }^{1}$ \\ Politecnico di Torino (PoliTo), Turin, Italy \\ P. Della Vecchia ${ }^{2}$ \\ Università di Napoli "Federico II" (UNINA), Naples, Italy \\ G. Donelli ${ }^{3}$ \\ German Aerospace Center (DLR), Institute of System Architectures in Aeronautics, Hamburg, \\ Germany \\ P. Hansmann ${ }^{4}$ \\ Rheinisch-Westfälische Technische Hochschule (RWTH), Aachen, Germany
}

\begin{abstract}
The integration of on-board systems design within the aircraft design process is often considered only in the late part of the initial design. This is acceptable for civil aircraft using standard technology systems. However, facing with MEA and AEA concepts and different possible architectures, the systems design and the assessment of their effects on the overall aircraft should be moved up in the usual design process. This paper deals with evaluation of the effect of different on-board systems architecture, with a different electrification level, on the overall aircraft design. These effects have been evaluated using three different MDA workflows developed within the AGILE4.0 European research project. The workflows are defined with an increasing number of disciplines to show how the effect of a proper selection of a systems architecture is differently caught by each one. In this way it is possible to define which disciplines should be included for the systems architecture assessment. The results show a save of $1 \%$ of MTOM for the AEA applied to a small turboprop aircraft when only the OBS mass is assessed. Increasing workflow complexity, adding performance and engine design the save increase to $1.2 \%$. Finally, the save increases to $1.3 \%$ when the effect on engine SFC is also considered.
\end{abstract}

$\begin{array}{ll}\text { ACM } & =\text { Air Cycle Machine } \\ \text { AEA } & =\text { All Electric Aircraft } \\ \text { ECS } & =\text { Environmental Control System } \\ \text { EHA } & =\text { Electro Hydrostatic Actuator } \\ \text { FCS } & =\text { Flight Control System }\end{array}$

\section{Nomenclature}

${ }^{1}$ Assistant professor, Department of Mechanical and Aerospace Engineering

${ }^{2}$ Assistant professor, Department of Aerospace Engineering

${ }^{3}$ Researcher, Department of Aircraft Design \& System Integration

${ }^{4}$ Researcher, Institute of Aerospace Systems 


$\begin{array}{ll}\text { IPS } & =\text { Ice Protection System } \\ \text { MDA } & =\text { Multidisciplinary Design Analysis } \\ \text { MDO } & =\text { Multidisciplinary Design Optimization } \\ \text { MEA } & =\text { More Electric Aircraft } \\ \text { MTOM } & =\text { Maximum Take Off Mass } \\ \text { OAD } & =\text { Overall Aircraft Design } \\ \text { OBS } & =\text { On-Board Systems } \\ \text { SFC } & =\text { Specific Fuel Consumption } \\ \text { TLARs } & =\text { Top Level Aircraft Requirements } \\ \text { TOFL } & =\text { Take Off Field Length }\end{array}$

\section{Introduction}

The majority of the MDA/MDO workflows are focused on airframe - aerodynamics - engine integrated design (1), (2), (3). The design of the On-Board Systems (OBS) is usually considered secondary for the design process of a civil transport aircraft and it is performed later in the design process. This could be considered a good compromise between design time and results accuracy when facing with standard OBS architecture which involve the use of hydraulic actuators, pneumatic Environmental Control System (ECS) and aerothermal Ice Protection System (IPS). Nowadays, the need for a more efficient and rational OBS lead to the electrification of flight control and landing gear actuation as well as the electrification of ECS and IPS (4), (5). This is also proven by the last civil aircraft such as Boeing 787, Airbus 380 and 350 which incorporate some of these new technologies. The presence of several possible OBS architectures, with different electrification level, forces to include the OBS design from the first steps of the aircraft design process. This is needed since the difference in terms of mass, power requirement and cost of each architecture is not negligible (6), (7), (8). Therefore, the MDA/MDO workflows that has been set up, with the aim to study a modern transport aircraft, should include a more detailed OBS design module (9), (10).

The present work has been carried out in the frame of the AGILE4.0 European research project which is focused on reducing aircraft development cost and time introducing new technologies and novel design disciplines to MDO problems such as aircraft certification, production and upgrade. The main technical objectives of the project are:

- Develop Technologies streamlining integration and collaboration in the supply chain;

- Develop solutions accelerating trade-off and decision making;

- A novel design and optimization Paradigm for complex systems;

- Implement a reconfigurable computational design environment.

The application case here discussed is developed in the scope of the "certification driven stream" that is a series of MDO problems involving different aspects of aircraft certification. They will range from safety analysis of aircraft sub-systems to the aircraft continuous airworthiness and its maintainability. In this way, the aircraft certifiability will be increased from the first phases of the design allowing a reduction of the development time and cost. As a preparatory work, the integrated design of a small regional aircraft and its OBS has been carried out. The aircraft and the architecture of its OBS will be the basis of future project steps which will embrace the presence of certification related disciplines.

In section III, the reference aircraft and the different OBS architectures are described and increasing electrification level are identified. In section IV, the results are reported emphasizing the effect on the OBS assessment adding and increasing number of disciplines to the MDA/MDO workflow. Finally, the conclusions have been drawn also highlighting the next expected developments. 


\section{Reference aircraft and OBS architectures}

The reference aircraft is a small twin turboprop, 19 passengers regional transport aircraft capable of $1500 \mathrm{~km}$ of maximum range. All the aircraft Top Level Aircraft Requirements are listed in Table 1.

Table 1. Reference aircraft TLARs

\begin{tabular}{|l|l|l|}
\hline & Metric & Imperial \\
\hline MTOM & $\leq 8600 \mathrm{~kg}$ & $\leq 19000 \mathrm{lb}$ \\
\hline PAX & $\leq 19$ & $\leq 19$ \\
\hline Range & $\leq 1500 \mathrm{~km}$ & $\leq 800 \mathrm{~nm}$ \\
\hline Speed & $0.45 \mathrm{M}$ & $0.45 \mathrm{M}$ \\
\hline Ceiling & $7600 \mathrm{~m}$ & $25000 \mathrm{ft}$ \\
\hline TOFL & $<800 \mathrm{~m}$ & $<2600 \mathrm{ft}$ \\
\hline
\end{tabular}

Four different OBS architectures have been considered for this kind of aircraft. Each of them has a different electrification level.

For this aircraft category, the conventional architecture is depicted in Fig. 1(a). The Flight Control System (FCS) is mechanical for primary surfaces and hydraulically actuated secondary surfaces (i.e. flaps). Considering the aircraft dimensions and speed, this is considered the best compromise in terms of performance and cost. The ECS is needed to pressurize the cabin and to control its intern temperature. The cabin pressurization is needed considering the ceiling altitude requirement. The ECS uses two standard 3-wheels Air Cycle Machine (ACM) pneumatically powered by the engine bleed system. The bleed air is also used to inflate and deflate the boots of the de-icing system that cover the leading edge of the wings and horizontal tail plane. An electrothermal de-icing system is considered for sensors, windscreens, propeller blades, engine intakes and the compensation horns of the movable control surfaces. The extraction/retraction, the steering and breaking of landing gears are hydraulically actuated. The conventional architecture needs a triple power system (i.e. pneumatic, hydraulic, and electric systems) each of one is configured as double lane. The electric system has two low voltage (i.e. 28 VDC) starter generators and two inverters to supply the $28 \mathrm{VDC}$ and $115 \mathrm{VAC} 400 \mathrm{hz}$ busses.

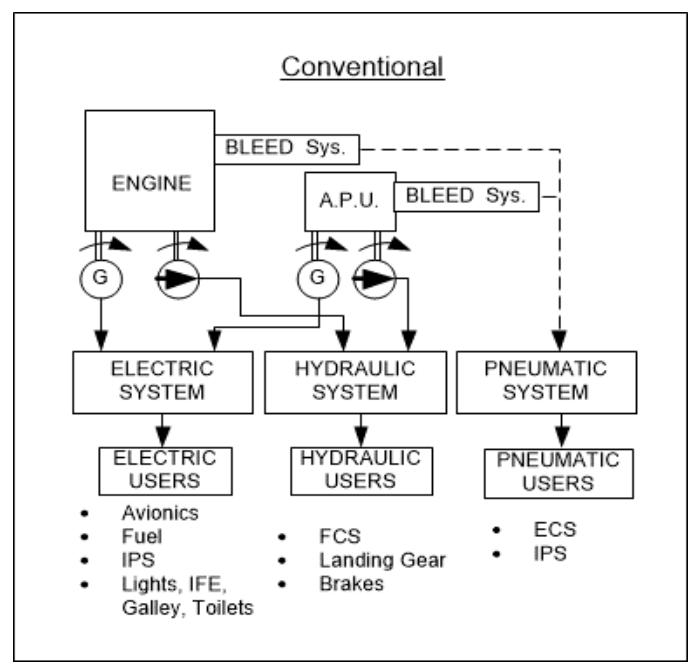

(a)

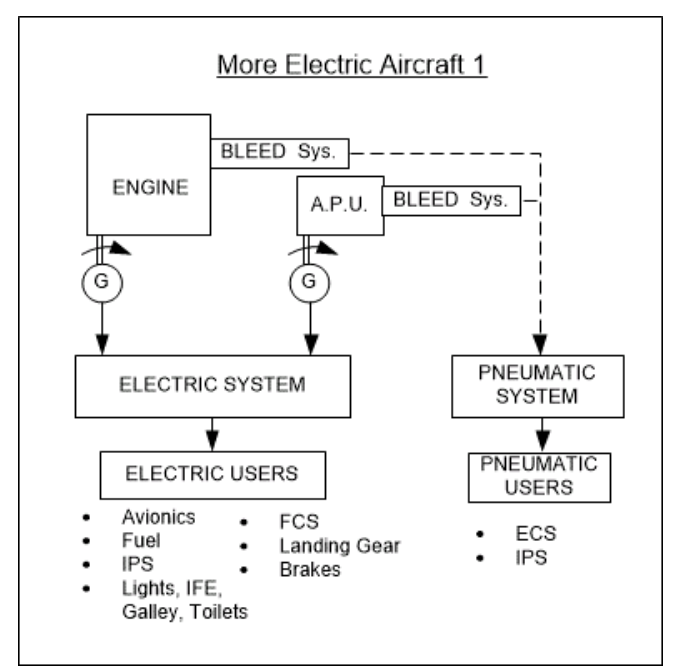

(b)

Fig. 1 Conventional (a) and more electric n.1 (b) OBS architectures.

The first more electric architecture (MEA1) (see Fig. 1(a)) is inspired to the last Airbus aircraft that use some electrical actuators replacing the hydraulic ones. In MEA1 all hydraulic system and users are removed. The flap and landing gear system hydraulic actuators are replaced by Electro Hydrostatic Actuators (EHA) supplied by a high voltage 
electric system. The electric system provides 270 VDC voltage by means of two starter generators. All electric users are connected to a double lane 270 VDC bass. The IPS and ECS are conventional and supplied by the pneumatic system using the engine bleed air.

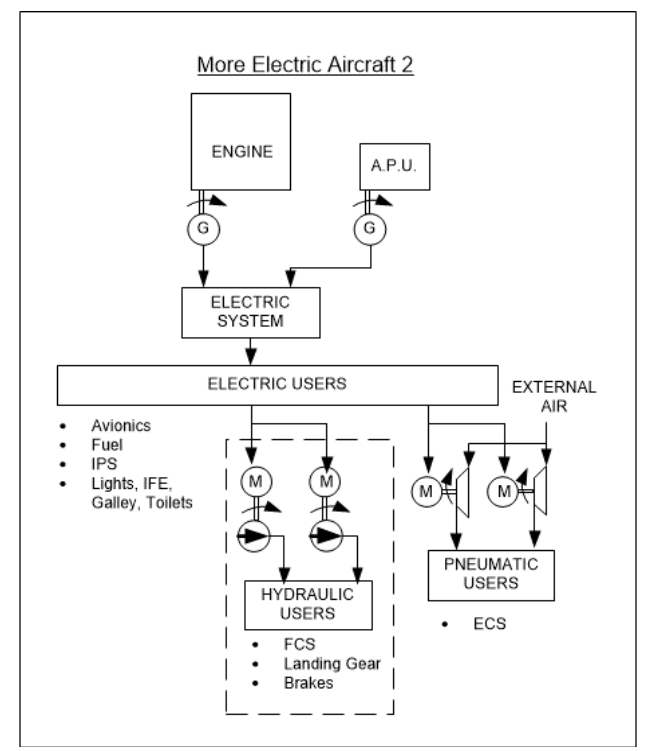

(a)

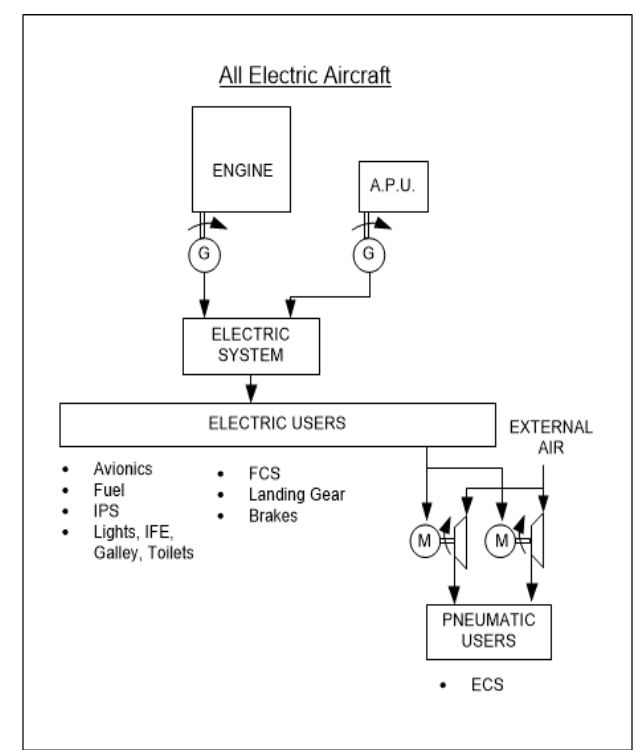

(b)

Fig. 2 More electric n.2 and all electric OBS architectures.

The second more electric architecture (MEA2) (see Fig. 2(a)) is inspired by Boeing 787 transport aircraft. In this case, the IPS and ECS has been electrified. All parts of the airplane needing ice removal are equipped with electrothermal de-icing system. The ECS is supplied by external air compressed by two centrifugal compressors driven by electric motors. In this way, the pneumatic and engine bleed systems are removed. The actuation system for flap and landing gear is conventional (i.e. hydraulic). However, the hydraulic pumps are driven by electric motors instead being mechanically connected to the engine gearbox. The electric system generates high voltage current (270 VDC) by means of two starter generators.

The all-electric architecture is depicted in Fig. 2(b). It incorporates all electrified systems of MEA1 and MEA2 architecture. Therefore, the flap and landing gear actuators are electrical as well as the IPS and ECS systems. The pneumatic and hydraulic systems are completely removed. The high voltage electric system is the only system dedicated to power generation and distribution.

\section{Implementation of the design workflows}

Three different workflows have been implemented with an increasingly level of integration and fidelity. The main aim is to explain how, for the case of OBS design, the integration of some disciplines is needed to entirely take into account for the effect of systems electrification on the whole aircraft. All workflow has been integrated within RCE MDAO environment (11) using CPACS (12) as connecting file to transfer the information among the tools.

The tools included in the workflows are:

- OpenAD (DLR). Overall aircraft conceptual design tool. Starting from aircraft TLARs, it is able to generate aircraft geometry, define main aircraft masses and their position.

- $\quad$ ASTRID (PoliTo). OBS integrated design tool. All main user systems are designed by ASTRID including Avionics, FCS, landing gear, IPS, ECS, fuel system and all power systems (i.e. pneumatic, hydraulic, and 
electric systems). The tool is based on semi-empirical and physic-based algorithms and it is capable to design more electric and all electric architectures.

- Performance (UNINA). Aircraft performance calculation tool. Several aircraft performance are calculated including flight envelope, balance field length, glide ratio, rate of climb, maximum ceiling, mission fuel, mission range and time.

- Engine (UNINA). Engine design tool. Based on scaled engine deck, it is able to calculate engine performance, main dimensions and mass.

- $\quad$ SFC_sensitivity (PoliTo). Engine performance tool. Based on scaled engine deck, this tool defines the new engine Specific Fuel Consumption (SFC) considering the systems power offtakes and bleed air requirements.

The first workflow proposed is a simple connection between the Overall Aircraft Design (OAD) and the OBS design. It is needed to assess the different OBS architecture separately to the other disciplines. The results of this workflow are used as reference to understand the effect of integrating other disciplines.

The second workflow proposed is depicted in Fig. 3. The OBS is here connected with performance and engine design disciplines. The mass variation due to the different OBS configuration produces aircraft with different mass. The performance tool is able to recalculate the mission fuel considering the new aircraft mass. It also produces new thrust requirements that are used by the engine tool to redesign the engine. The engine is redesigned in terms of performance, dimensions and mass. Then a loop assures aircraft mass convergency. Using this workflow only the effect of OBS mass variation is taken into account.

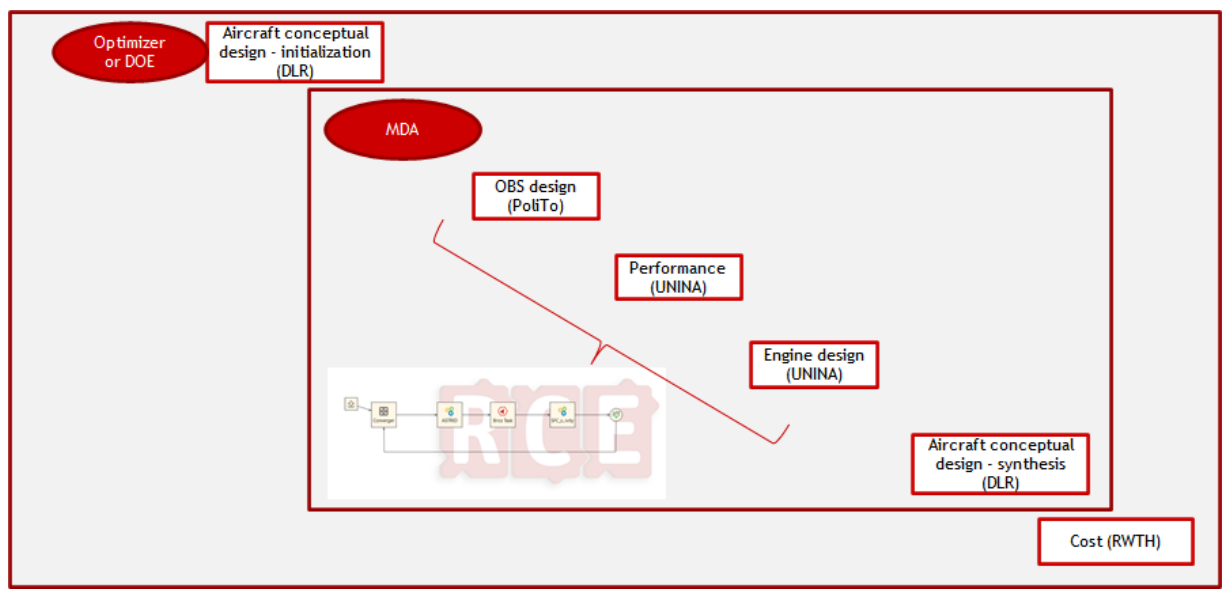

Fig. 3. Second workflow tested.

The third workflow (see Fig. 4) includes the SFC_sensitivity tool among the other tools already mentioned for the second workflow. The SFC_sesitivity is able to modify the engine SFC according to the OBS power offtakes and bleed requirements. The new engine SFC is then acquired by engine tool which is able to calculate new values of fuel flow and, by means of performance tool, new mission fuel mass. This last workflow has the capability to caught both the effect on engine SFC and aircraft mass due to the different OBS architectures. 


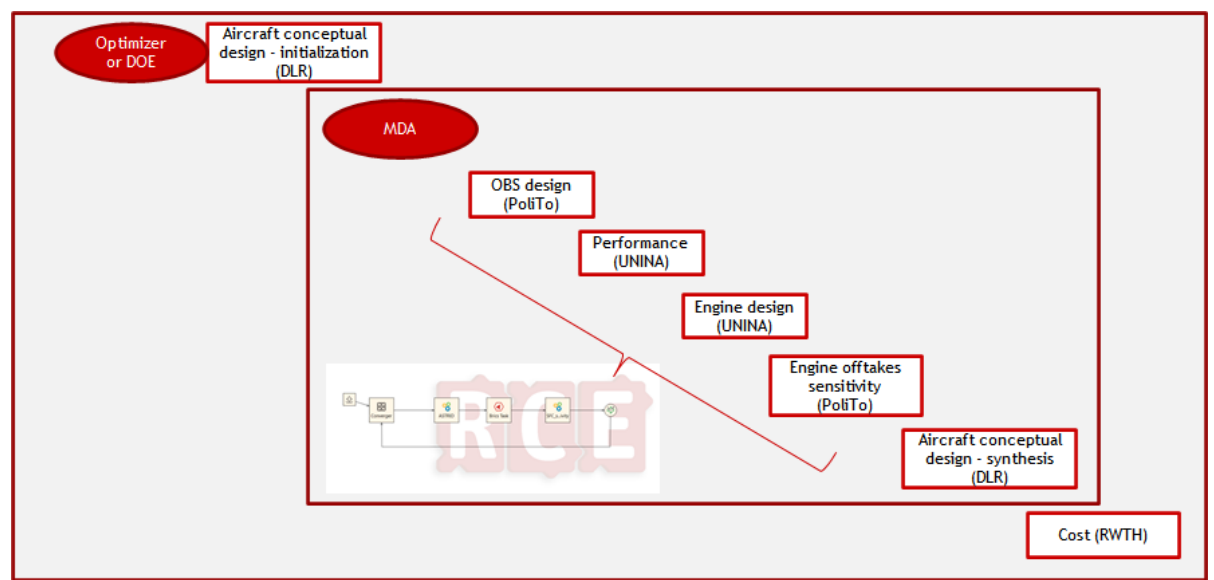

Fig. 4. Third workflow tested.

\section{Results of the MDA workflows}

Before focusing on the different results obtained running the three workflows, the results of OAD and performance are showed. Starting from the aircraft TLARs, OAD generates the aircraft geometry depicted in Fig. 5 and the main specification listed in Table 2. The aircraft configuration is equivalent to similar aircraft already produced. The wing position have been analyzed to obtain static stability. The fuselage is elliptical considering the need for pressurization. The aircraft is compliant with the initial requirements showing an MTOM of about 8.5 tons, a TOFL of $800 \mathrm{~m}$, a ceiling of $7620 \mathrm{~m}$ and maximum range of $1200 \mathrm{~km}$.

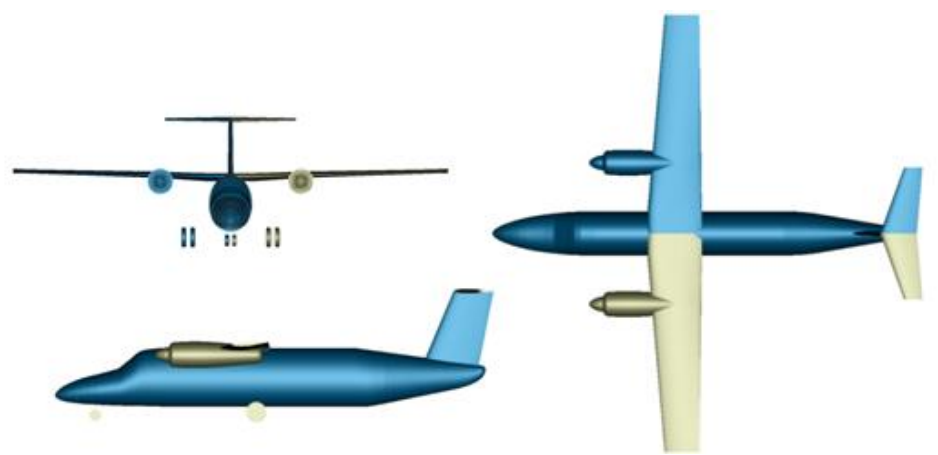

Fig. 5. Three views of the reference aircraft.

Table 2. Specification of the reference aircraft.

\begin{tabular}{|c|c|c|}
\hline \multicolumn{3}{|l|}{ Specifications } \\
\hline $\mathrm{N}$ pax & 19 & - \\
\hline Mass per pax & 83 & $\mathrm{~kg}$ \\
\hline Max Payload & 1985 & $\mathrm{~kg}$ \\
\hline Design Range & 650 & $\mathrm{~nm}$ \\
\hline Design Payload & 1767 & $\mathrm{~kg}$ \\
\hline Design Cruise Mach & 0.45 & - \\
\hline sTOFL & 800 & $\mathrm{~m}$ \\
\hline Cruise Altitude & 7620 & $\mathrm{~m}$ \\
\hline N Pilots & 2 & - \\
\hline MTOM & 8532 & $\mathrm{~kg}$ \\
\hline OEM & 5509 & $\mathrm{~kg}$ \\
\hline MZFW & 7494 & $\mathrm{~kg}$ \\
\hline
\end{tabular}


The results of OAD are also confirmed and refined by the performance tool. From the flight envelope depicted in Fig. 6(a), it is worth noting the aircraft reaches (and slightly exceed) the required speed at ceiling altitude. This is the design point for the propulsion system. For this reason, as shows in Fig. 6(b), the aircraft outperform the takeoff field length requirement. Therefore, the engines could be flat rated for ground operation. Moreover, the speed requirement lead to a greater real ceiling altitude as depicted in Fig. 7(a). However, as for other aircraft (e.g. Beechcraft 1900d), the maximum ceiling is limited by the cabin pressurization at $7600 \mathrm{~m}$. This decrease the fuselage mass since there is no need for this kind of aircraft to flight at higher altitude. Finally, Fig. 7(b) shows the aircraft meet the maximum range requirement of $1200 \mathrm{~km}$.

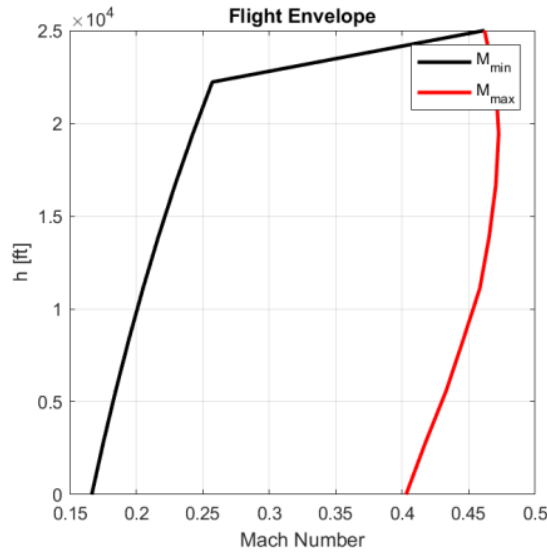

(a)

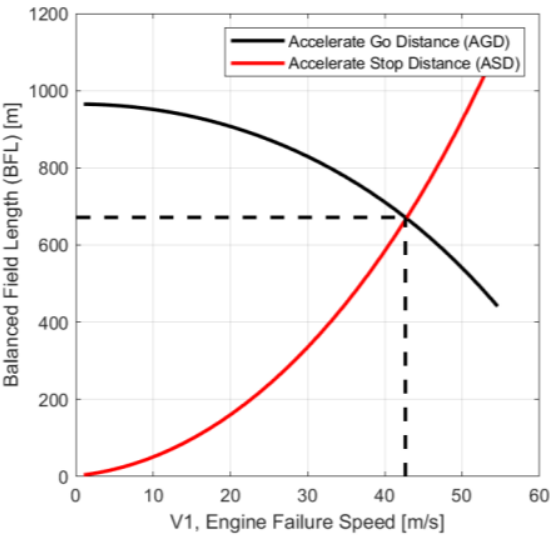

(b)

Fig. 6. Aircraft envelope and balanced field length.

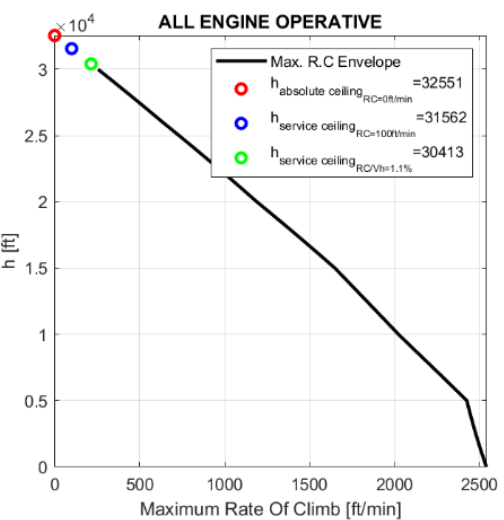

(a)

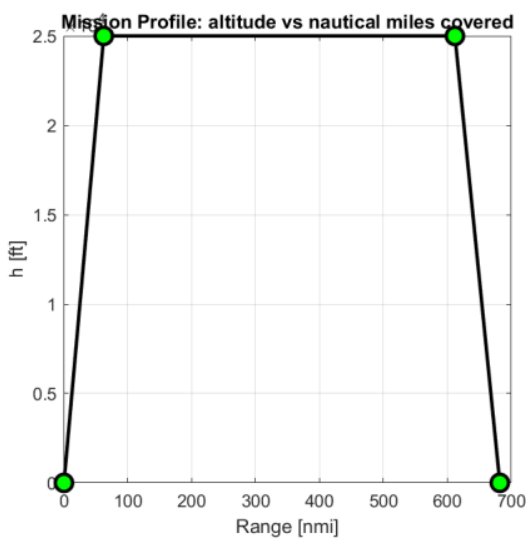

(b)

Fig. 7. Rate of climb and mission profile.

The main aircraft masses and the mass of the four OBS architectures are summarized in Table 3 . All the architectures have been analyzed using each workflow being the aim of the paper to address, gradually, the effect of OBS design on the other disciplines. Focusing on the mass of each systems, it is worth noting that very slight difference exists when comparing the results of the different workflows. This proves that the addition of other disciplines does not substantially modify the design of the OBS since a more detailed aircraft performance and engine design quite 
aligned with the OAD results do not directly affect the OBS. However, this strongly depends on the disciplines included. As instance, adding RAMS (Reliability, Availability, Maintainability and Safety) analysis would have a greater impact on OBS architectures and their masses (13).

Table 3. Results of the three MDA workflow

\begin{tabular}{|c|c|c|c|c|c|c|c|c|c|c|c|c|}
\hline \multicolumn{4}{|c|}{ Workflow n.1 } & \multicolumn{5}{|c|}{ Workflow n.2 } & \multicolumn{4}{|c|}{ Workflow n.3 } \\
\hline & Conv & MEA1 & MEA2 & AEA & Conv & MEA1 & MEA2 & AEA & Conv & MEA1 & MEA2 & AEA \\
\hline MTOW $[\mathrm{kg}]$ & 8549 & 8443 & 8525 & 8459 & 8553 & 8429 & 8524 & 8448 & 8571 & 8448 & 8533 & 8457 \\
\hline $\mathrm{ZFW}[\mathrm{kg}]$ & 7509 & 7402 & 7484 & 7418 & 7495 & 7381 & 7469 & 7398 & 7503 & 7389 & 7475 & 7404 \\
\hline OEM [kg] & 5524 & 5417 & 5499 & 5433 & 5510 & 5396 & 5484 & 5413 & 5518 & 5404 & 5490 & 5419 \\
\hline M_FUEL [kg] & 1041 & 1041 & 1041 & 1041 & 1058 & 1049 & 1055 & 1050 & 1068 & 1059 & 1058 & 1053 \\
\hline $\begin{array}{r}\text { Operator items } \\
{[\mathrm{kg}]} \\
\end{array}$ & 468 & 468 & 468 & 468 & 468 & 468 & 468 & 468 & 468 & 468 & 468 & 468 \\
\hline \begin{tabular}{|l|} 
Tot sys mass [kg] \\
\end{tabular} & 1520 & 1414 & 1496 & 1429 & 1511 & 1397 & 1485 & 1414 & 1512 & 1400 & 1488 & 1414 \\
\hline & & & & & & & & & & & & \\
\hline $\begin{array}{r}\text { Avionic_mass } \\
{[\mathrm{kg}]}\end{array}$ & 135 & 135 & 135 & 135 & 135 & 135 & 135 & 135 & 135 & 135 & 135 & 135 \\
\hline FCS_mass [kg] & 142 & 145 & 142 & 145 & 141 & 143 & 141 & 144 & 142 & 144 & 142 & 144 \\
\hline IPS_mass [kg] & 71 & 71 & 76 & 76 & 71 & 71 & 76 & 76 & 71 & 71 & 76 & 76 \\
\hline ECS_mass [kg] & 107 & 107 & 129 & 129 & 107 & 107 & 129 & 129 & 107 & 107 & 129 & 129 \\
\hline Fuelsys_mass [kg] & 34 & 34 & 34 & 34 & 27 & 27 & 27 & 27 & 27 & 26.6 & 26.6 & 27 \\
\hline LNDG_mass [kg] & 336 & 358 & 336 & 358 & 335 & 353 & 334 & 353 & 336 & 354 & 335 & 353 \\
\hline $\begin{array}{r}\text { Furnishing_mass } \\
{[\mathrm{kg}]} \\
\end{array}$ & 379 & 379 & 379 & 379 & 379 & 377 & 378 & 378 & 379 & 377 & 378 & 378 \\
\hline APU_mass [kg] & 0 & 0 & 0 & 0 & 0 & 0 & 0 & 0 & 0 & 0 & 0 & 0 \\
\hline $\begin{array}{r}\text { Pneumatic_mass } \\
{[\mathrm{kg}]}\end{array}$ & 56 & 56 & 0 & 0 & 56 & 56 & 0 & 0 & 56 & 56 & 0 & 0 \\
\hline $\begin{array}{r}\text { HYD_sys_mass } \\
{[\mathrm{kg}]}\end{array}$ & 97 & 0 & 92 & 0 & 97 & 0 & 92 & 0 & 97 & 0 & 92 & 0 \\
\hline EL_sys_mass [kg] & 164 & 129 & 174 & 174 & 164 & 129 & 174 & 174 & 164 & 129 & 174 & 174 \\
\hline
\end{tabular}

Focusing on the systems masses, some trends are notable. The electrification of the flap and landing gear actuation increase their masses since the greater mass of the EHA compared to the hydraulic one. Comparable outcome can be noted when the ECS has been electrified. In this case, the electric-driven dedicated compressors are specifically added to supply the ECS and their mass is considered as part of the ECS mass. However, when the overall OBS mass is considered, the electrification produces a beneficial effect. Firstly, the electrification of the actuation system leads to the removal of hydraulic system as well as the electrification of the ECS and IPS leads to the removal of the pneumatic system. Secondly, the electrification rationalizes the power supply for the systems increasing the amount of electrical power. This leads to the opportunity to increase the voltage level reducing the electric system mass. This effect is notable when comparing the electric system mass of the conventional with the MEA1 architecture.

Considering the total OBS mass, the MEA1 is the best architecture followed by AEA, MEA2 and conventional. The use of high voltage electric system and the removal of the hydraulic system produce the more beneficial effect on OBS mass. The removal of the pneumatic system is counterbalanced by the increment of the electrified ECS mass disadvantaging the MEA2 architecture. This result is also confirmed at aircraft level when the aircraft MTOM is considered. 


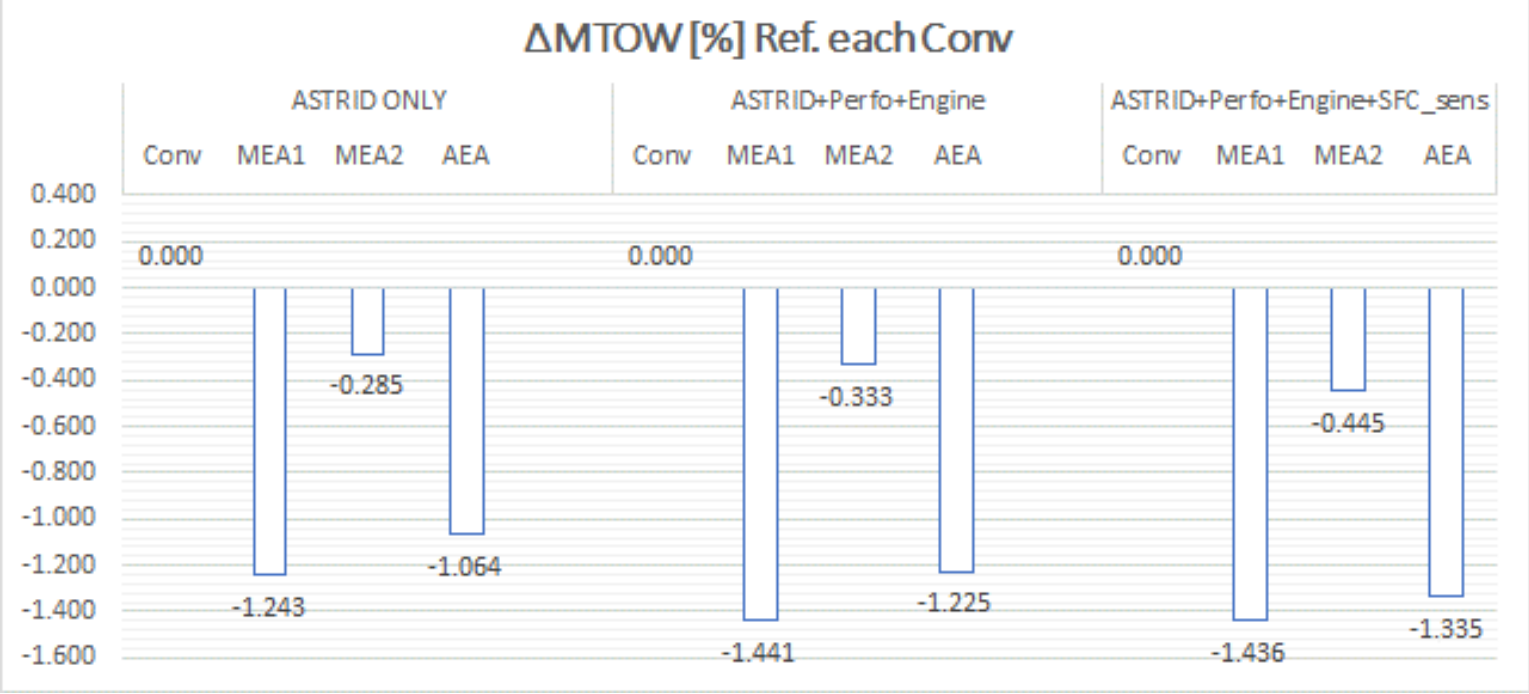

Fig. 8. Different assessment of OBS architectures using the three workflows under study.

The Fig. 8 shows the relative difference of the electrified OBS architectures, in terms of MTOM, having as reference the aircraft MTOM of the conventional architecture. This comparison has been depicted for each workflow. It is worth noting that the save of about $1.2 \%$ of MTOM could represent the mass of one additional passenger for a 19-pax aircraft. Comparing the results of the different workflows, the mass savings increases when additional disciplines are considered. This means that the second workflow is able to consider the mass snowball effect on the engine mass and fuel mass due to the reduced OBS mass. The third workflow adds a further characteristic of the electrified architectures: the effect on engine SFC. The removal of the need for engine bleed air (for MEA2 and AEA architectures) produces a beneficial effect reducing the engine SFC thus reducing the mission fuel. The results of the third workflow show a further increase of the mass saving for the bleedless architectures (i.e. MEA2 and AEA). Instead of MEA1 that remains stable compared with the results of the second workflow since no significant effect is produced on engine SFC.

\section{Conclusion}

The paper confirms the importance of evaluating all main effect of OBS design when the design of different architectures is needed. The effect of different mass and power offtakes have to be considered to correctly compare the architectures. The electrified architectures are defined following the real trend of last civil transport aircraft and the results support it. The electrification of all actuators together with the removal of the hydraulic system (i.e MEA1 architecture) produce a sensible reduction of aircraft mass. On the other hand, the electrification of the ECS and IPS (MEA2 architecture) leads to a bleedless configuration enhancing engine efficiency and reducing the fuel burnt.

However, to correctly assess both the effects, the MDAO workflow needs to evaluate the snowball effect on the overall aircraft, provide an engine design sensible to the different systems offtakes and calculate the aircraft performance thus the new fuel required. A simpler workflow produces under evaluated and deceptive results that could lead to a selection of a sub-optimal architecture.

Nevertheless, the third workflow proposed is capable to correctly assess the effect on OAD but some disciplines directly affecting OBS design are still missing. As instance, the safety analysis and aircraft certifiability directly affect the definition of the OBS architecture requiring different number of lanes, equipment redundancy and alternate performance. Therefore, the future steps of the AGILE4.0 will include the safety analysis integrated to the workflow as well as a thermal risk assessment of some highly loaded comportments such as the electronic bay. Finally, in order to assess the best OBS architecture a cost estimation module will be added.

\section{Acknowledgments}


The research presented in this paper has been performed in the framework of the AGILE 4.0 project (Towards cyber-physical collaborative aircraft development) and has received funding from the European Union Horizon 2020 Programme under grant agreement no 815122.

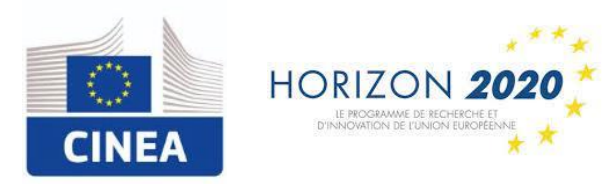

\section{References}

1. Martins Pires RM, Lajux V, Fielding JP. Methodology for the Design and Evaluation of Wing Leading Edge and Trailing Edge Devices. In International Congress of the Aeronautical Sciences; 2006; Hamburg, Germany.

2. Sobieszczanski-Sobieski J. Multidisciplinary Design Optimization: An Emerging New Engineering Discipline. Advances in Structural Optimization. 1995;: p. 483-496.

3. Werner-Westphal C, Heinze W, Horst P. Multidisciplinary Integrated Preliminary Design Applied to Unconventional Aircraft Configuration. Journal of Aircraft. 2008; 45(2): p. 581-590.

4. Cronin MJ. All-Electric vs Conventional Aircraft: The Production/Operational Aspects. Journal of Aircraft. 1983; 20(6): p. 481-486.

5. Sinnet M. 787 No-Bleed Systems: Saving Fuel and Enhancing Operational Efficiencies. Aero Quarterly QTR_04 |07. 2007;: p. 06-11.

6. Vecchia PD, Stingo L, Nicolosi F, De Marco A, Cerino G, Ciampa PD, et al. Advanced turboprop multidisciplinary design and optimization within agile project. In 2018 Aviation Technology, Integration, and Operations Conference; 2018: American Institute of Aeronautics and Astronautics Inc, AIAA.

7. Torrigiani F, Bussemaker J, Ciampa PD, Fioriti M, Tomasella F, Aigner B, et al. Design of the strut braced wing aircraft in the agile collaborative MDO framework. In 31st Congress of the International Council of the Aeronautical Sciences; 2018.

8. Fioriti M, Vercella V, Viola N. Cost-Estimating Model for Aircraft Maintenance. AIAA Journal of Aircraft. 2018; 55(4): p. 1564-1575.

9. Chakraborty I, Mavris DN. Integrated Assessment of Aircraft and Novel Subsystem Architectures in Early Design. AIAA SciTech. 2016.

10. Fioriti M, Boggero L, Prakasha PS, Mirzoyan A, Aigner B, Anisimov K. Multidisciplinary aircraft integration within a collaborative and distributed design framework using the AGILE paradigm. Progress in Aerospace Sciences. 2020 Nov 1.

11. Official RCE Webpage. [Online]. Available from: http://rcenvironment.de/.

12. Common Language for Aircraft Design. [Online].; 2019 [cited 2019 May. Available from: http://cpacs.de.

13. Chiesa S, Fioriti M. UAV logistic support definition. In Handbook of Unmanned Aerial Vehicles.: Springer Netherlands; 2015. p. 2565-2600. 